\title{
Étude d'une grotte sépulcrale préservée de Nouvelle-Calédonie
}

\section{Christophe Sand et André-John Ouetcho}

\section{(2) OpenEdition \\ 1 Journals}

Édition électronique

URL : http://journals.openedition.org/jso/6172

DOI : $10.4000 /$ jso.6172

ISSN : 1760-7256

\section{Éditeur}

Société des océanistes

\section{Édition imprimée}

Date de publication : 15 décembre 2010

Pagination : 221-228

ISBN : 978-2-85430-027-7

ISSN : 0300-953x

\section{Référence électronique}

Christophe Sand et André-John Ouetcho, «Étude d'une grotte sépulcrale préservée de NouvelleCalédonie », Journal de la Société des Océanistes [En ligne], 130-131 | 2010, mis en ligne le 15 décembre 2010, consulté le 24 septembre 2020. URL : http://journals.openedition.org/jso/6172 ; DOI : https:// doi.org/10.4000/jso.6172

\section{(C) Tous droits réservés}




\section{MISCELLANÉES}

\section{Étude d'une grotte sépulcrale préservée de Nouvelle- Calédonie}

par

\section{Christophe SAND* et André-John OUETCHO*}

Si les traditions orales kanak et les descriptions des premiers explorateurs occidentaux qui ont sillonné l'archipel calédonien décrivent parfois de façon détaillée l'existence de grottes sépulcrales renfermant de nombreux objets (voir par exemple Lambert, 1900), les multiples pillages et ramassages occasionnés par les visiteurs de passage ont, depuis le $\mathrm{XIX}^{\mathrm{e}}$ siècle, progressivement vidé ces sites anciens de leur contenu mobilier, quand ce n'est pas également de leurs restes osseux. Dans ce contexte, la découverte et l'étude préliminaire d'une grotte sépulcrale apparemment intacte ouvre une fenêtre sur ce que devaient être, avant le processus colonial, les dépôts funéraires intacts. Ce texte présente les données obtenues sur un site de la Grande Terre calédonienne (province Nord), à la suite du signalement en 2001 par un spéléologue amateur d'une grotte isolée, renfermant des vestiges archéologiques ${ }^{1}$. Une première visite avait alors permis de confirmer que ce site était particuliè- rement riche en vestiges anciens et comportait des indications claires d'utilisation comme lieu de sépulture, incitant à réaliser en avril 2003 une étude préventive du site, avant d'en fermer l'entrée afin d'éviter des pillages.

\section{La grotte}

La grotte sépulcrale est localisée dans un environnement karstique. Elle mesure, à partir de son unique entrée, $19 \mathrm{~m}$ dans un axe sud/sudouest - nord/nord-est et $29 \mathrm{~m}$ dans un axe estouest (partie centrale) (figure 1). Le dénivelé entre le sol de l'entrée et le sol du fond de la grotte centrale est de $2,8 \mathrm{~m}$ (axe sud-nord), la hauteur de plafond variant entre 0,5 et $2 \mathrm{~m}$, avec une moyenne de $1,5 \mathrm{~m}$. Les stalactites ont toutes été cassées à une date ancienne puisque des fistuleuses, pouvant atteindre plusieurs centimètres, ont eu le temps de se reformer. Une

1. Pour des raisons évidentes de protection, l'emplacement exact du site n'est pas indiqué dans cette note. En plus de l'enregistrement dans les archives de l'Institut d'archéologie de la Nouvelle-Calédonie et du Pacifique (IANCP), la position du site a été déposée auprès de la direction de la culture de la province Nord et remise aux autorités coutumières concernées, afin de garder une trace au cas où, dans le futur, des recherches plus approfondies apparaîtraient nécessaires.

* Institut d'archéologie de la Nouvelle-Calédonie et du Pacifique (IANCP), christophe.sand@iancp.nc et andre-john. ouetcho@iancp.nc 

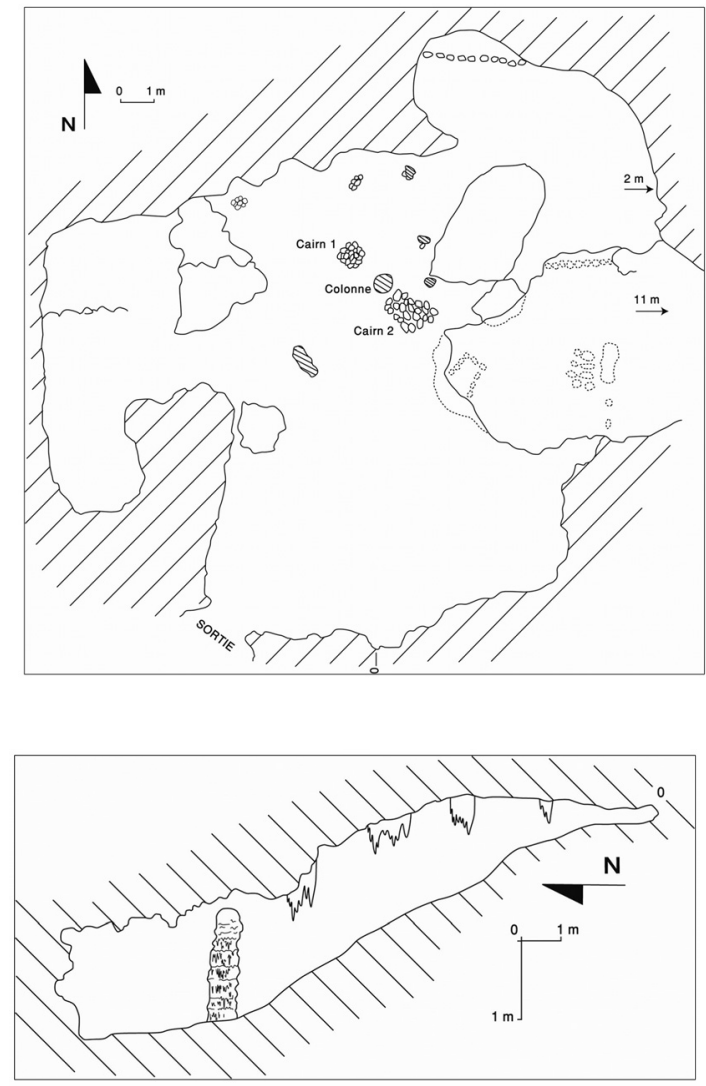

Figure 1. - Plan général de la grotte sépulcrale fermée et coupe de la partie centrale de la grotte sépulcrale (à partir du point 0 du plan) chambre centrale d'environ $80 \mathrm{~m}^{2}$ est flanquée à l'ouest d'une petite pièce d'environ $30 \mathrm{~m}^{2}$ et à l'est d'un boyau se poursuivant sur près de $15 \mathrm{~m}$. Ces deux ensembles latéraux sont en partie en forte déclivité et présentent une faible hauteur de moins de 1,2 m. Différents décrochements, avec dalles et conduits, subdivisent chaque zone. Le sol est formé d'un remplissage rougeâtre, composé de sédiments issus de la décomposition du corail, de terres infiltrées de la surface et du guano produit par les oiseaux.

\section{Les structures}

La pièce centrale concentre la grande majorité des vestiges archéologiques, en particulier dans la partie centrale nord (figure 2). Le point focal est formé par une stalagmite d'environ $150 \mathrm{~cm}$ de hauteur et $70 \mathrm{~cm}$ de diamètre moyen, en forme d'obus, dont le sommet est entièrement piqueté et comporte des traces de raclage. Il n'est pas évident que cette stalagmite soit à sa place naturelle de formation. Il est plus probable qu'elle ait été placée là par l'action humaine, mais seule une fouille à sa base permettrait de confirmer cette hypothèse. Au nord-ouest de cette colonne se trouve un cairn composé d'une accumulation de blocs de corail fossile et de stalactites brisées

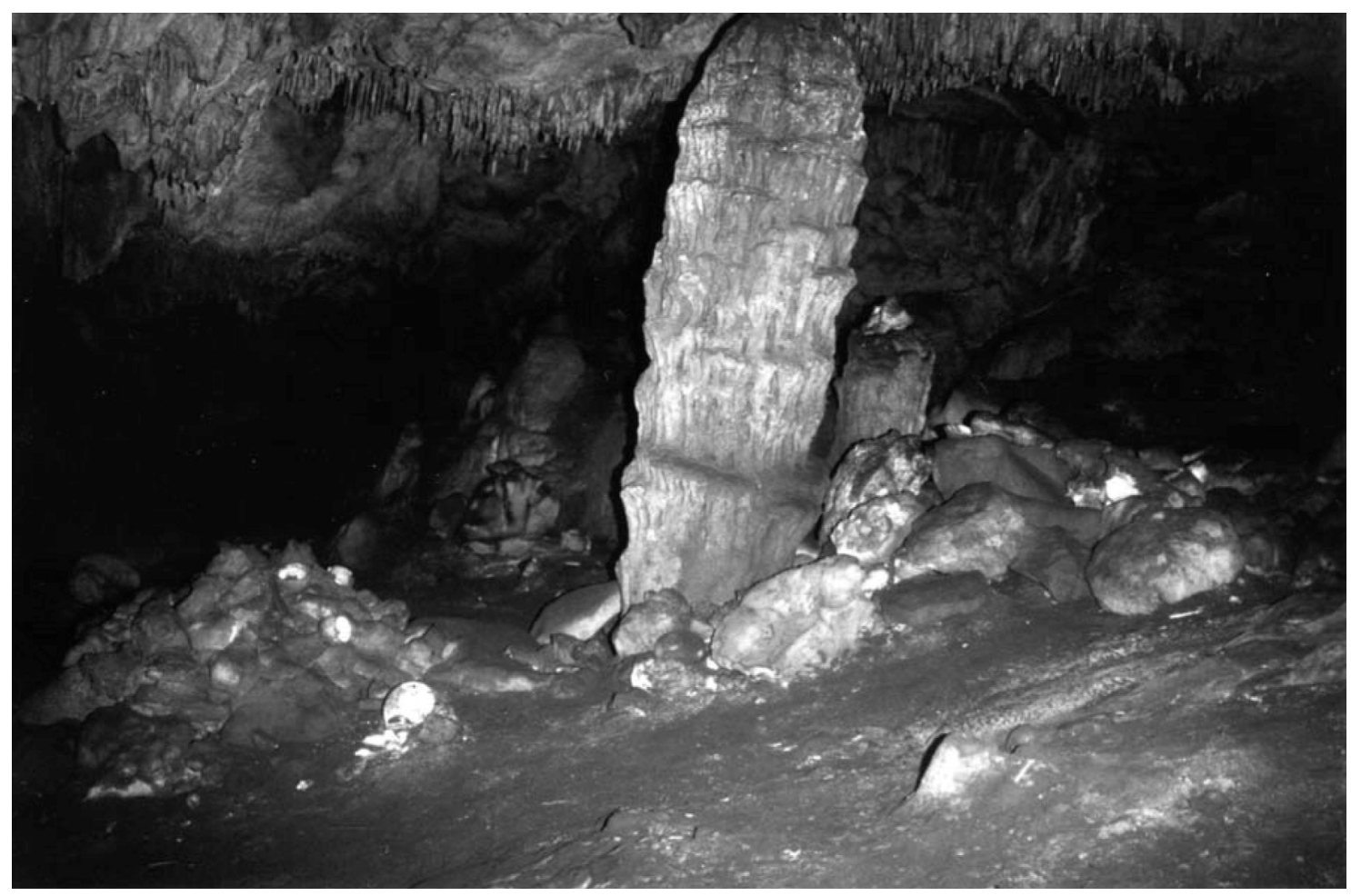

FIGURE 2. - Vue de la partie centrale de la grotte funéraire, avec la colonne et les deux cairns principaux 


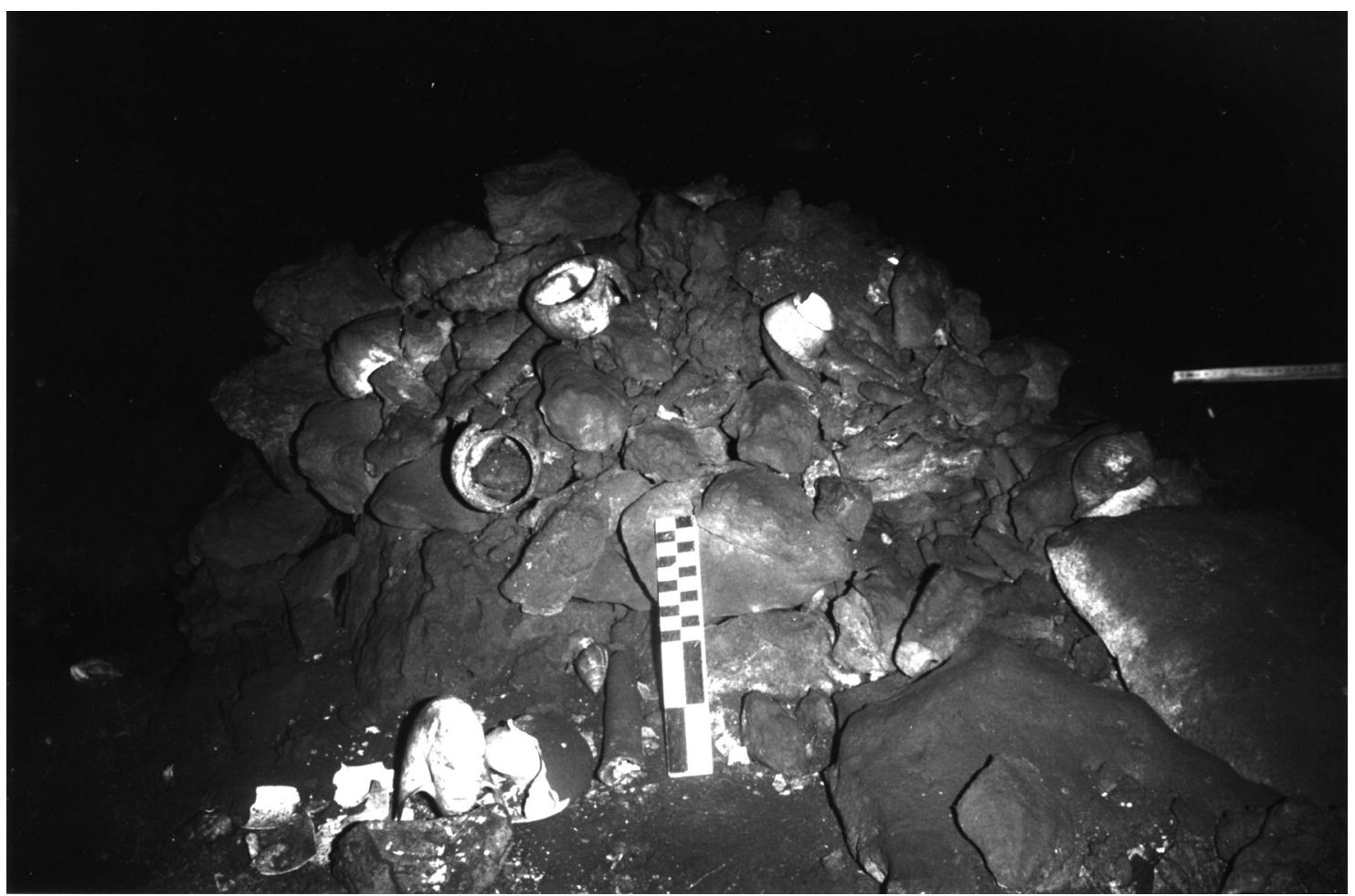

FIGURE 3. - Vue du cairn 1, renfermant au moins un crâne humain, avec les bracelets en cône posés en sommet et des nautiles à la base

(cairn 1), de forme grossièrement rectangulaire et mesurant $70 \mathrm{~cm}$ de côté sur 40 de hauteur. Un crâne humain est visible au centre de cette structure (figure 3).

$\mathrm{Au}$ sud-est, un autre cairn de plus grande dimension (130 cm sur 120 dans sa plus grande largeur) a une hauteur maximale de $60 \mathrm{~cm}$ (cairn 2). Aucun ossement humain n'est visible entre les pierres, mais on note la présence de blocs de roches minérales (fer et chrome) dans l'appareillage du cairn. Un de ces blocs ferreux est très compact et lourd. D'autres blocs rocheux d'origine péridotique sont présents autours du cairn. Ces roches ne sont pas naturellement présentes dans l'environnement karstique où se trouve le site. Ils ont donc été apportés par l'homme dans la grotte après avoir été transportés sur une distance de plusieurs kilomètres minimum. Une petite stalagmite, cassée, est située à l'est de la colonne centrale et mesure $80 \mathrm{~cm}$ de hauteur : un coquillage (porte-montre) percé a été placé sur son sommet.

Un autre ensemble d'aménagements se trouve à la limite de la pièce centrale, au début du boyau est. Sous une grosse dalle effondrée ont été localisées deux niches basses renfermant des squelettes humains bordés par un petit entourage de blocs de corail fossile. Le choix de dépôt funéraire laisse à penser qu'ils ne sont pas néces- sairement de même époque que les os du cairn ou que ces défunts avaient un statut social différent. Le sommet d'un autre alignement de blocs de corail fossile se distingue à l'entrée du boyau, en partie recouvert naturellement par du sédiment. Quelques ossements humains ont également été observés dans le boyau ouest: certains de ceux-ci pourraient être issus d'un glissement de sol provenant de la surface. D'autres petits cairns sont présents dans les parties profondes de la grotte.

\section{Objets archéologiques}

Cinquante-cinq objets ont été observés dans la zone immédiatement autours de la colonne centrale et une trentaine d'autres dans les différents compartiments latéraux (hors ossements humains et tesson). Une visite relativement récente, qui n'apparaît pas avoir donné lieu à un pillage, est prouvée par la découverte d'une bougie au pied de la colonne. Il doit être noté que différents objets étaient, lors de l'étude, partiellement ou presque complètement enfouis dans le sol, indiquant que d'autres vestiges se trouvent probablement aujourd'hui en stratigraphie, sous la surface du sol. 
TABLEAU 1 - Famille de coquillage et répartition (* = Bracelet non terminé)

\begin{tabular}{|c|c|c|c|c|c|}
\hline \multirow[t]{2}{*}{ Catégorie } & \multicolumn{2}{|c|}{ Autour de la colonne } & \multicolumn{2}{|c|}{ Compartiments latéraux } & \multirow[t]{2}{*}{ Total } \\
\hline & Percé & Non percé & Percé & Non percé & \\
\hline 1. Turbo sp. & 0 & 0 & 0 & 3 & 3 \\
\hline 2. Strombus sp. & 0 & 1 & 0 & 2 & 3 \\
\hline 3. Nautilus macrophalus & 0 & 15 & 0 & 6 & 21 \\
\hline 4. Trochus niloticus & 0 & 3 & 1 & 1 & 5 \\
\hline 5. Porte-montre & 1 & 3 & 2 & 1 & 7 \\
\hline 6. Charonia tritonis (triton) & 1 & 1 & 0 & 2 & 4 \\
\hline 7. Lambis sp. & 1 & 2 & 0 & 1 & 4 \\
\hline 8. Ovula ovum (porcelaine blanche) & 1 & 1 & 0 & 0 & 2 \\
\hline 9. Tonna sp. & 5 & 5 & 2 & 3 & 15 \\
\hline 10. Bursidae (?) & 1 & 2 & 0 & 0 & 3 \\
\hline 11. Bracelet en Conus sp. & 0 & 7 & 0 & $1^{*}$ & 8 \\
\hline 12. Placostylus sp. & 0 & 1 & 0 & 3 & 4 \\
\hline 13. Tridacnidae & 0 & 2 & 0 & 1 & 3 \\
\hline 14. Pecten sp. (coquille St-Jacques) & 1 & 1 & 0 & 0 & 2 \\
\hline Total & & & & & 84 \\
\hline
\end{tabular}

L'étude a permis de relever quatorze catégories différentes d'objets présents à plus d'un exemplaire (tableau 1). Parmi les coquillages comportant des indications claires de transformation anthropique, se trouvent des bracelets en trocas et en cône, ainsi que des tritons, des porcelaines blanches, des porte-montres et des Bursidae volontairement percés. Il faut ajouter à ces objets une râpe dentée en nacre, un couteau en nacre et un os de seiche, ainsi que plusieurs coquilles d'Anadara scapha. Un seul tesson, fin, de type tradition de Balabio ( $1^{\mathrm{er}}$ millénaire après J.-C.) a été découvert au pied du cairn 1. La figure 4 positionne les objets découverts dans la grotte, avec une concentration autour de la colonne centrale. L'étude de répartition permet de noter qu'il n'y a pas réellement de concentrations des différentes catégories d'objets suivant l'emplacement dans le site, mais plutôt une diversité entre les trois aires de répartition que sont la colonne et les deux cairns.

Les coquillages (à l'exception possible des Placostylus, dont au moins deux variétés semblent présentes) ont tous été apportés volontairement dans la grotte. La majorité comporte des traces de transformation pour en faire des objets utiles : trous de ligature, percement pour être enfilés ou servir de conque, polissage d'un bord pour créer un tranchant, etc. Certaines pièces sont clairement des parures, comme les bracelets en cône et les " coquilles de chef» (Bursidae) percées. D'autres ont probablement servi de conques d'appel ou étaient à un moment donné enfilées sur une perche. La présence de nombreuses coquilles de nautile, coquillage par nature fragile, est peut-être liée à une utilisation spécifique («symbolique»?) de ce céphalopode. La figure 5 présente quelques exemples d'objets inventoriés.

\section{Premières datations}

Afin d'avoir une indication générale de la période de mise en place d'au moins une partie de ce site, des datations ont été réalisées, l'une sur du charbon prélevé en surface dans le début du boyau est et l'autre sur une coquille d'Anadara prélevée en surface à la base de la colonne centrale. Après traitement, le charbon a été daté à deux sigmas de 520 (400) 380 avant J.-C. (Beta179507) et la coquille de 350 (440) 540 après J.-C. (Beta-179508). Ces datations viennent compléter le corpus d'informations disponibles à ce stade pour le site, bien qu'il faille souligner que l'interprétation de ces résultats reste difficile car aucun des deux échantillons ne provient de contextes stratigraphiques contrôlés.

\section{Discussion}

L'étude réalisée sur l'abri funéraire découvert en 2001 a permis de se faire une idée relativement précise du contexte existant jusqu'à la colonisation sur la Grande Terre dans ce type de site, avant que la grande majorité d'entre eux ne soit pillée, afin en particulier de récupérer les parures en coquillage. Au vu du nombre de vestiges présents en surface, dans une grotte finalement de 

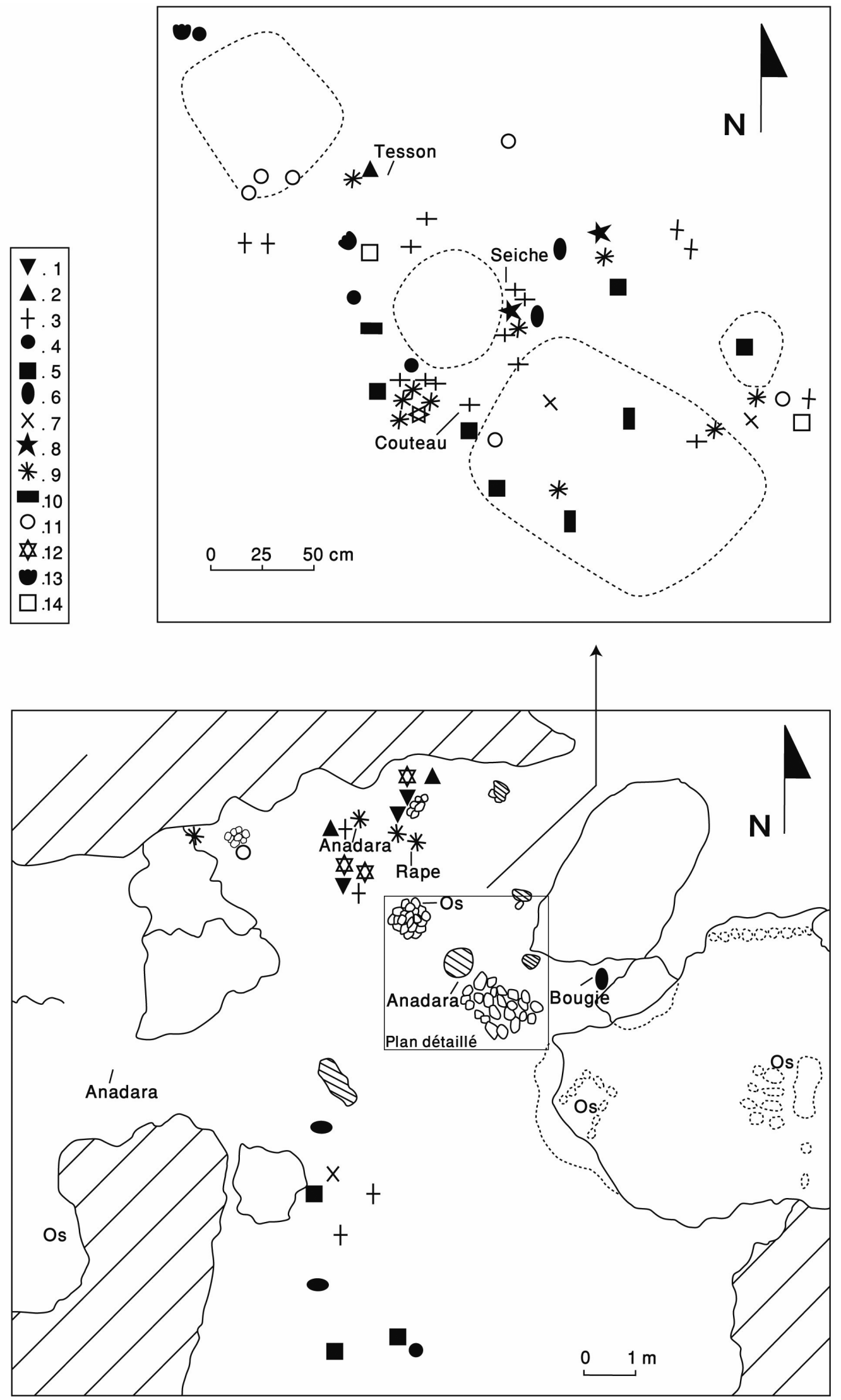

FIGURE 4. - Répartition des vestiges archéologiques dans le reste de la salle principale, avec le détail des vestiges autour de la colonne centrale (se référer aux numéros du tableau 1) 

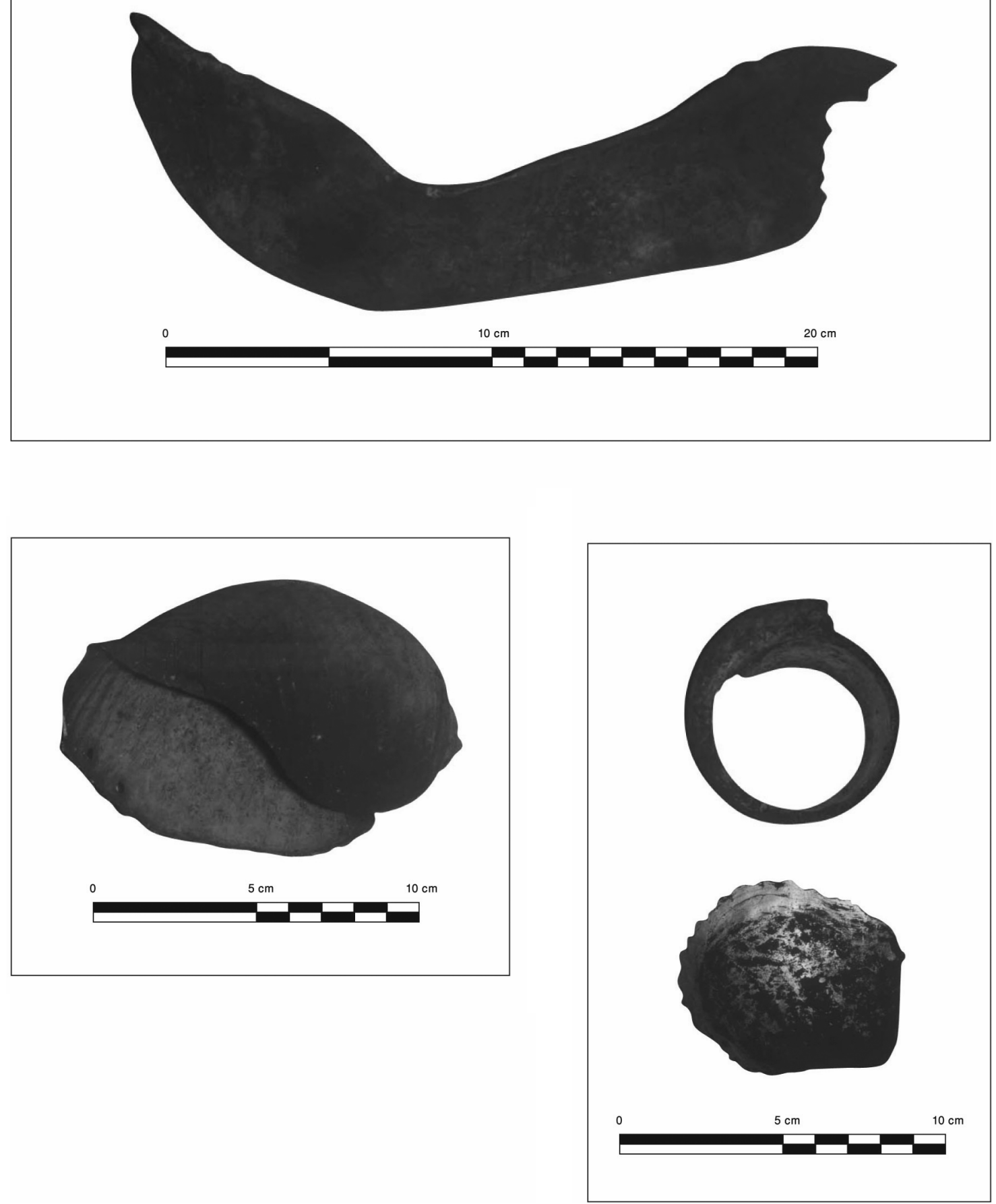

FIGURE 5. - Différents types d'objets présents dans la grotte sépulcrale

petite taille et sans même la réalisation de fouilles, on comprend mieux l'immense quantité de parures en coquillage issues de l'archipel, présentes dans les collections muséographiques et privées.

La diversité et le manque apparent d'ordre dans le positionnement des objets autour de la colonne rendent difficile toute interprétation directe de ce site. Le caractère funéraire de la grotte est démontré par la présence de sépultures à entourage de blocs de corail et par l'observation d'au moins un crâne dans le cairn 1. Mais la présence sur la colonne centrale - qui forme de toute évidence le point focal de l'organisation spatiale de la grotte - de nombreuses traces de coups et de quelques traces de polissage/raclage 
pouvant indiquer que cet aménagement avait une fonction " rituelle », démontre une utilisation de la cavité ne se limitant pas à l'aspect funéraire. On sait en effet que certains rituels océaniens impliquent l'écrasement de fibres végétales (Leenhardt, 1937).

L'absence de vestiges osseux apparents entre les blocs du grand cairn 2, contrairement au cairn 1, ainsi que l'utilisation de blocs rocheux nécessairement apportés volontairement de loin pour l'aménagement de la structure laissent à penser que, s'il ne s'agit pas d'une sépulture sans os visibles, cette structure plus étendue aurait pu être une sorte de reposoir ou d'autel plutôt qu'une tombe. Faute de fouilles, il n'est également pas possible de définir l'utilisation des petits cairns présents dans la grotte, bien que leur taille réduite rende une utilisation comme sépulture d'adulte difficilement imaginable, laissant à penser que ces structures devaient avoir une autre finalité. La présence de plusieurs « coquilles de chef» (Bursidae) ainsi que d'un couteau en nacre et d'une lame en Lambis est peut-être une indication d'une utilisation spécifique de cet aménagement formant le cairn 2. D'autres objets ont peut-être été bougés, en particulier lors des visites plus récentes faites dans la grotte. Ainsi, le porte-montre placé sur la petite stalagmite cassée a peut-être été mis à sa place actuelle bien après la période d'utilisation du site pour des rituels. De même, il n'est pas évident que les trois bracelets placés sur le cairn 1 soient dans leur position initiale. Il doit être noté que d'autres cairns de même forme et ayant probablement eu des utilisations diverses (sépultures, espaces à rituels) ont été identifiés dans d'autres grottes proches du site décrit ici. Il ne s'agit donc pas d'un type d'aménagement exceptionnel pour la région, cette tradition étant par ailleurs bien connue des sociétés océaniennes, en particulier sur la Grande Terre (Leenhardt, 1937).

L'absence de fouilles dans la grotte et l'impossibilité de dater directement les objets et les ossements dans le cadre de l'étude de 2003, puisqu'aucune pièce n'a été emportée pour étude détaillée, rendent toute définition de la période de mise en place de cet ensemble funéraire, difficile. Au vu des deux datations réalisées sur des échantillons de surface, il apparaît néanmoins que les structures présentes dans l'abri remontent au minimum entre la fin du premier millénaire avant $\mathrm{J} .-\mathrm{C}$ et le premier millénaire après J.-C., une chronologie renforcée par la présence d'un tesson apparenté à la tradition de Balabio découvert en surface. Une datation indirecte par les fistuleuses formées sur les stalactites brisées volontairement, apparaît confirmer cette hypo- thèse d'une utilisation relativement ancienne de la grotte.

\section{Conclusion}

La présentation synthétique d'une étude de grotte funéraire de la Grande Terre calédonienne non pillée a permis de montrer la complexité probable d'utilisation de ce type de site dans le passé. Outre l'aspect simplement funéraire, peutêtre lui-même divisé en au moins deux époques distinctes (sous cairn et en niche), la grotte a été de toute évidence utilisée pour des rituels de nature indéfinie, identifiables en particulier par la présence de surfaces écrasées et raclées en sommet de la colonne centrale, placée volontairement entre les deux cairns principaux. Les objets découverts en surface des structures se rattachent quant à eux à une typologie déjà connue pour la Grande Terre. Dans ce contexte, il doit être souligné que les deux datations obtenues pour le moment indiquent une chronologie précédant l'émergence de l'ensemble culturel traditionnel kanak et l'apparition de certains de ses objets (Sand, 2001), venant encore renforcer l'hypothèse d'une utilisation du site à plusieurs périodes de la chronologie calédonienne.

À l'issue de la cartographie et de l'étude des vestiges de surface, la grotte funéraire a été fermée, en prenant soin de permettre la poursuite de la circulation de l'air à l'intérieur de la cavité. Cette découverte exceptionnelle a été l'occasion pour l'ancien département Archéologie de Nouvelle-Calédonie de mener une information auprès du public calédonien à travers les médias, afin de sensibiliser la population de l'archipel sur la nécessité de protéger ce type de patrimoine fragile. Un relevé photographique détaillé et une série de dessins ont été faits pour archive, afin de servir de base à une étude complète de ce site unique dans l'inventaire archéologique calédonien.

\section{Remerciements}

Cette étude a été menée à bien dans le cadre des activités de l'ancien département Archéologie de Nouvelle-Calédonie pour le compte de la province Nord. Les autorisations préalables ont été obtenues auprès des autorités coutumières de la zone du site. Nous souhaitons remercier le découvreur Laurent Lemaire, pour nous avoir contactés et avoir accepté de nous montrer la grotte, et Stéphanie Domergue pour la PAO des plans. 


\section{BIBLIOGRAPHIE}

LAMBERT révérend père, 1900. Mours et superstitions des Néo-calédoniens, Nouméa, Nouvelle Imprimerie nouméenne.

LEENHARDT Maurice, 1937. Gens de la Grande Terre, Paris, éditions Gallimard (republié en version augmentée en 1953).
SAND Christophe, 2001. Changes in non-ceramic artefacts during the prehistory of New Caledonia, in $\mathrm{G}$. Clark, T. Sorovi-Vunidilo and A. Anderson (eds), The archaeology of Lapita dispersal in Oceania (Papers from the Fourth Lapita Conference, June 2000, Canberra, Australia), Canberra, Australian National University, RSPAS, Occasional Papers in Prehistory, pp. 75-92. 